\section{Effects of Plantar Flexor Muscles Fatigue on Postural Control during Quiet Stance and External Perturbation in Healthy Subjects}

\author{
Rojhani-Shirazi Z. ${ }^{1,2}$, Amiri Z. ${ }^{1,2}$, Ebrahimi S. ${ }^{1,2 *}$
}

\begin{abstract}
Background: The maintenance of postural control is a key component in dynamic physical activity, especially during muscle fatigue and against external forces. Despite many studies in this field, there is no consensus regarding the effects of plantar flexor muscles fatigue on postural control during different postural tasks.

Objective: To evaluate the effects of plantar flexor muscles fatigue on postural control during quiet stance and external perturbation in healthy subjects.

Material and Methods: Twenty four healthy individuals (20-35 years) participated this interventional study. The foot center of pressure data was measured using a single force platform, and then the postural control parameters, including the center of pressure displacement and velocity in the anterior-posterior and medial-lateral direction and also path length calculated under two conditions; quiet and perturbed stance, before and after plantar flexor muscles fatigue.

Results: The statistical analysis demonstrated that mean displacement and velocity of the center of pressure in the anterior-posterior direction and also path length increased after the fatigue protocol in the perturbed condition. However, fatigue had no significant effects on postural control parameters in the quiet standing condition.

Conclusion: These results indicated that the effects of muscle fatigue on postural control depend on the difficulty of the task and the relevance of proprioceptive information. The postural control system appears to use distinct control strategies in different situations such as quiet and perturbed stance conditions, and these strategies may be differentially altered by fatigue. In conclusion, due to the potential risk of loss of balance, it is important to take the role of plantar flexor muscle fatigue into account during more difficult postural tasks.
\end{abstract}

\section{Keywords}

Center of Pressure, Perturbation, Fatigue, Plantar flexor, Balance

\section{Introduction}

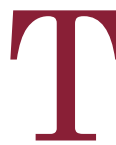
he ability to maintain stable upright stance is vital in sports and daily living activities. To this end, the postural control system must integrate and reweight information received from the visual, vestibular, and somatosensory systems, and must often react quickly in response to an external perturbation [1]. These perturbations result from external forces and torques elicited by rapid changes in the distribution of body mass, which may lead to losing the balance and increasing the incidence of falling [2]. Ankle response is sufficient to
${ }^{1}$ Physical Therapy

Department, School of

Rehabilitation Sciences,

Shiraz University of

Medical Sciences, Shi-

raz, Iran

${ }^{2}$ Rehabilitation Sciences

Research Center, Shiraz

University of Medical Sci-

ences, Shiraz, Iran

Corresponding author:

S. Ebrahimi

Physical Therapy Department, School of Rehabilitation Sciences, Shiraz

University of Medical

Sciences, Shiraz, Iran

E-mail: Ebrahimis@

sums.ac.ir

Received: 10 February 2017 Accepted: 17 June 2017 
counteract minor perturbations during normal stance, and it is believed that in unperturbed conditions, ankle proprioception is one of the primary mechanisms for establishing an internal reference to organize and plan motor responses [3]. Previous studies have shown that the plantar flexor muscles play a significant role in postural corrections, especially in the sagittal plane [4-7]. Moreover, it has been shown that bipedal stance control is impaired when proprioceptive information from the ankle joints is degraded by vibratory stimulation [3], an unstable or moving support surface [8] or ankle muscle fatigue [9].

Muscle fatigue is common during strenuous dynamic physical activities. In order to understand the mechanism of fatigue, it is important to determine the type of fatigue generations involved central or peripheral. Peripheral factors in fatigue generation mainly include metabolic inhibition of the contractile process and excitation-contraction coupling failure. On the other hand, central fatigue is one of the primary limiting factors in sustained contractions, and is associated with decreased recruitment of new motor units, reduced firing frequency of the motor units, or both [10-12]. It has been reported that muscle fatigue may affect neuromuscular performance. Typical fatigue-related changes in performance include a decline in maximal torque or power output, altered afferent proprioceptive inputs, altered central processing of proprioception, and decreased ability to respond to perturbations [13-15].

Several studies have shown that ankle plantar flexor muscles fatigue can impair postural control during static standing $[6,9,16,17]$. However, some studies have shown that exercise inducing fatigue in these muscles had no effects on postural control [18-20], and some research concluded that ankle plantar flexor muscles fatigue impairs postural control only when visual information is removed [21]. Overall, in spite of the many studied carried out in this field, there is no consensus regarding the effects of plantar flexor muscles fatigue on postural control. Furthermore, existing studies on plantar flexor muscles fatigue have focused somewhat narrowly on the effects of external perturbation on postural control. Since perturbation and plantar flexor muscles fatigue conditions can separately alter the maintenance of upright stance [3], we hypothesized that muscle fatigue combined with postural perturbation might lead to even larger increases in postural sway. Therefore, the present study was designed to investigate the sudden effects of plantar flexor muscles fatigue on postural control during quiet standing and after a sudden unexpected external perturbation.

\section{Material and Methods}

\section{Participants}

Twenty four healthy female students at Shiraz University of Medical Sciences participated in this study (mean age: $24.8 \pm 2.9$ years; mean body weight: $54.8 \pm 5.3 \mathrm{~kg}$; mean height: $163 \pm 4.3 \mathrm{~cm})$. The participants were excluded if they had any history of surgery in their trunks and lower limbs, motor problems, neurological diseases, deformity in the spines or lower limbs and vestibular impairment. The current research was conducted at the Rehabilitation Sciences Research Center, Shiraz University of Medical Sciences, and Shiraz, Iran. A detailed explanation of the procedure was provided and all the participants signed an informed consent form approved by the university ethics committee.

\section{Experimental Procedure}

At first, the maximal voluntary isometric contraction (MVC) of the ankle plantar flexor muscles was determined with a digital dynamometer $\left(\mathrm{MIE}^{\circledR}\right.$ Ltd., Leeds, UK). Three maximum isometric contractions of the plantar flexors were measured and the largest isometric plantar-flexion contraction value was recorded as the MVC. Then, the foot center of pressure (COP) data was collected using a single force plate $\left(\right.$ Kistler $^{\circledR}$, Winterthur, Switzer- 
land) and QTM software (Proreflex, Qualisys Track Manager ${ }^{\circledR}$ Ltd., Sweden) at a sampling rate of $120 \mathrm{~Hz}$ under four different conditions: pre-fatigue static standing, pre-fatigue perturbed standing, post-fatigue static standing and post-fatigue perturbed standing. Each participant was tested in 8 trials (each of the four conditions twice) in upright stance and in each trial the participant stood barefoot on the force platform. The order of the static and perturbed conditions was varied randomly among participants. Each trial lasted 30 seconds with a rest period of at least 60 seconds between two consecutive trials.

Pre-fatigue

In the static condition, the participants maintained bipedal stance for 30 seconds on the force plate with their eyes open. They were then instructed to keep their arms at their sides, stand straight with their feet shoulderwidth apart and look directly ahead. This test was done twice.

In the perturbed condition, the participants stood on the force plate with their eyes open, their gaze directly ahead, their right shoulder in 90 degrees of flexion and their right palm upward. The participants kept their left arm relaxed at their side. A unidirectional force delivered rapidly and unexpectedly by the experimenter to the subjects' right side at shoulder level (on the right palm), as a load from a fixed distance away from the subjects' right hand. The weight of the applied load was normalized to $2.5 \%$ of each individual's weight. The load was held $20 \mathrm{~cm}$ above the participant's right palm and released suddenly in an unspecified time between 1 to 10 seconds. To standardize the experimental conditions, the participant and the experimenter were separated by a dark curtain so that participants would not know when the load would be delivered (Figure 1). Each trial lasted 30 seconds with a rest period of at least 60 seconds between two consecutive trials.

Fatigue Protocol

During the plantar flexor muscle fatigue pro-

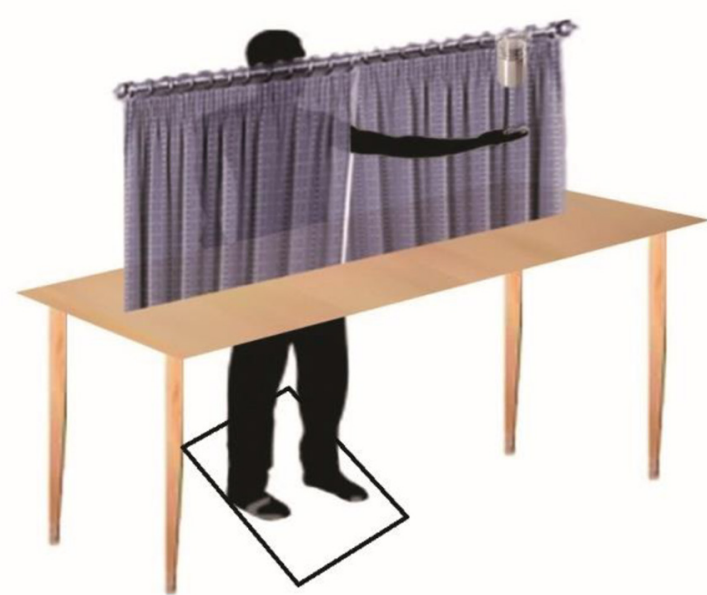

Figure 1: Schematic representation of subjects position during applying the external perturbation.

tocol, the participants sat on a bed with their hips and legs extended in front of them and their trunks upright at a 90-degree angle. One end of the dynamometer strap was fastened to a fixed bar behind them, and the other end was looped around the soles of both their feet (Figure 2). Once MVC was established, the subjects were instructed to look at a graph on a monitor and maintain $60 \%$ of their MVC

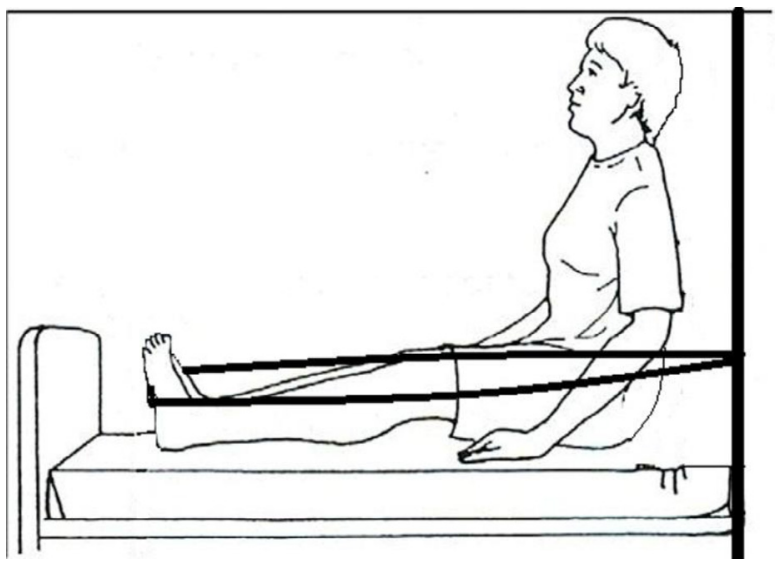

Figure 2: Schematic representation of subjects position during applying the fatigue protocol. 
by contracting their plantar flexor muscles to stretch the dynamometer strap. The fatigue protocol was stopped (i.e., the plantar flexor muscles were assumed to be fatigued) when the participants were unable to continue the task or when they no longer maintained the target degree of contraction and could not resume the intended force level in 5 seconds. The time between the fatigue protocol and the postural trials ( $<7$ minutes) was assumed to be shorter than the time needed for full recovery [22].

\section{Post-fatigue}

Immediately after the fatigue protocol, the tests in static and perturbed standing conditions were carried out as in the pre-fatigue protocol.

\section{Data analysis}

The COP data were used to calculate five postural control variables: mean medial-lateral (ML) COP displacement (mm), mean ML COP velocity, mean anterior-posterior (AP) COP displacement (mm), mean AP COP velocity $(\mathrm{mm} / \mathrm{s})$, and mean COP path length.

It should be stated that the AP and ML coordinates of COP were derived from the filtered data ( $4^{\text {th }}$ order Butterworth low-pass filter with a cut-off frequency of $10 \mathrm{~Hz}$ ) using Visual 3D motion analysis software (C-Motion ${ }^{\circledR}$, Rockville, MD, USA).

\section{Statistical Analysis}

The statistical analyses were done by SPSS software (version 21). One-way analysis of variance (ANOVA) with repeated measures was used for both factors, i.e. the two postural conditions (static vs. perturbed standing) and the two fatigue conditions (pre vs. post). Paired t-tests were used to compare COP parameters before and after the fatigue protocol in trials of static and perturbed standing. A P-value of $<0.05$ was considered statistically significant.

\section{Results}

The statistical analysis demonstrated that the AP COP displacement $(p=0.01)$, COP path length $(p<0.001)$ and AP COP velocity $(p<0.001)$ were significantly greater after plantar flexor muscles fatigue in the perturbed condition (Table 1, Figure 3A, 4, 5A). However, no significant differences were found in ML COP displacement $(\mathrm{p}=0.08)$ and ML COP velocity $(p=0.15)$ after plantar flexor muscle fatigue in the perturbed condition (Table 1, Figure 3B, 5B). Moreover, no significant changes were observed in postural control parameters during the static condition (Table 1, Figures 3-5). The statistical analysis showed that the changes in ML and AP displacement, path length and AP velocity after the fatigue protocol differed significantly between the static and perturbed conditions (Table 2).

Table 1: The comparison of mean postural control parameters before and after plantar flexor muscles fatigue in the static and perturbed standing conditions (mean \pm SD).

Dependent measures

Static standing

Perturbed standing

\begin{tabular}{cccccccc}
\hline & Before fatigue & After fatigue & P-value & Before fatigued & After fatigue & P-value \\
\hline ML displacement $(\mathrm{mm})$ & $2.84 \pm 0.70$ & $2.62 \pm 0.65$ & 0.17 & $3.89 \pm 0.56$ & $4.18 \pm 0.62$ & 0.08 \\
\hline AP displacement $(\mathrm{mm})$ & $1.64 \pm 0.79$ & $1.74 \pm 0.71$ & 0.34 & $2.90 \pm 0.71$ & $3.45 \pm 0.79$ & $0.01^{*}$ \\
\hline Path length $(\mathrm{m})$ & $21.89 \pm 1.62$ & $22.24 \pm 1.60$ & 0.07 & $23.40 \pm 1.64$ & $25.87 \pm 1.55$ & $<0.001^{*}$ \\
\hline ML velocity $(\mathrm{mm} / \mathrm{s})$ & $4.68 \pm 1.06$ & $4.56 \pm 1.42$ & 0.59 & $6.37 \pm 1.22$ & $6.84 \pm 1.40$ & 0.15 \\
\hline AP velocity $(\mathrm{mm} / \mathrm{s})$ & $3.54 \pm 0.79$ & $3.87 \pm 1.00$ & 0.15 & $5.48 \pm 1.01$ & $6.89 \pm 1.16$ & $<0.001^{*}$
\end{tabular}

ML: medial-lateral, AP: anterior-posterior, *Significant at $\mathrm{p}<0.05$ 


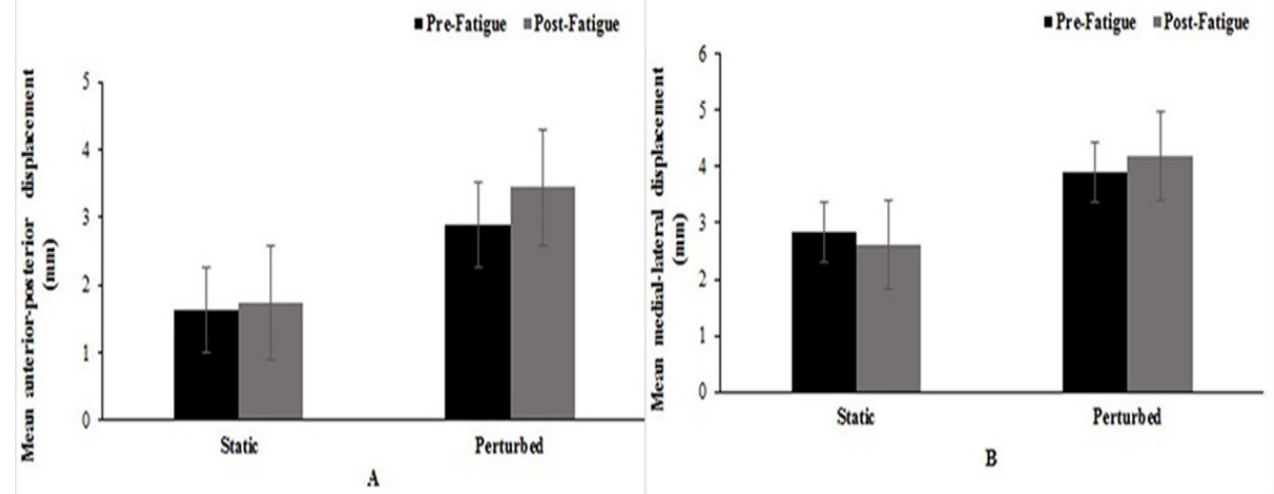

Figure 3: Mean anterior-posterior (A) and medial-lateral (B) displacement ( $\mathrm{mm}$ ) during static and perturbed standing conditions before and after plantar flexor muscle fatigue.

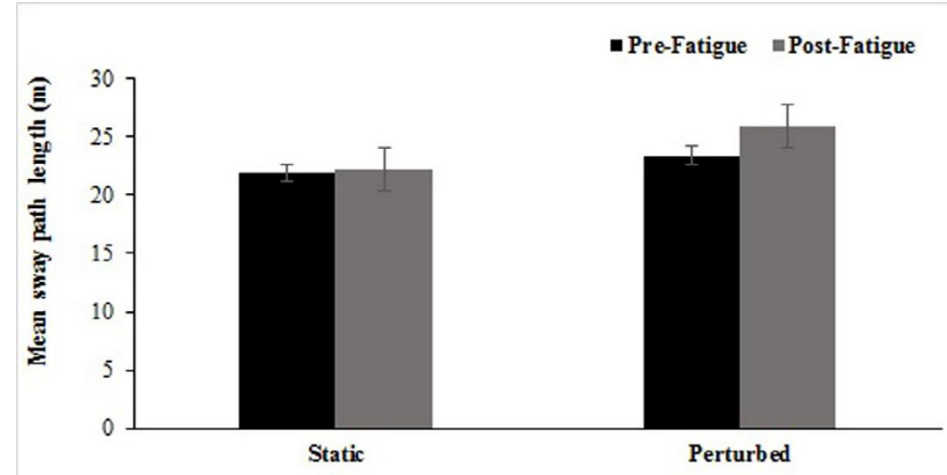

Figure 4: Mean sway path length $(\mathrm{m})$ during static and perturbed standing conditions before and after plantar flexor muscle fatigue.

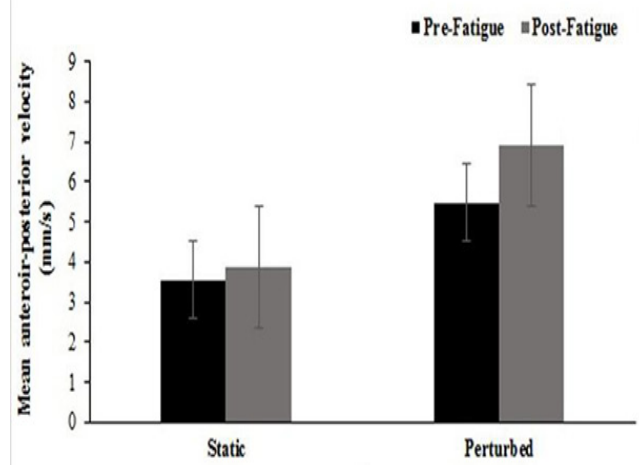

A

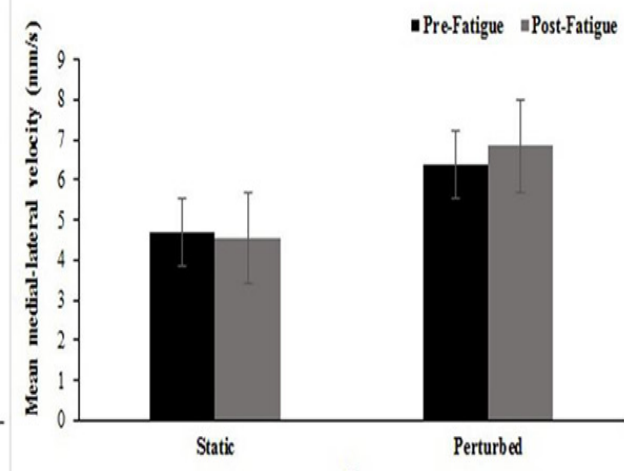

B

Figure 5: Mean anterior-posterior (A) and medial-lateral $(B)$ velocity $(\mathrm{mm} / \mathrm{s})$ during static and perturbed standing conditions before and after plantar flexor muscle fatigue. 
Table 2: The comparison of mean postural control parameters differences (after fatigue-before fatigue) between the static and perturbed conditions.

\begin{tabular}{cccc}
$\begin{array}{c}\text { Dependent } \\
\text { measures }\end{array}$ & \multicolumn{3}{c}{$\begin{array}{c}\text { Differentiation } \\
\text { value comparison }\end{array}$} \\
\hline & Static diff & Perturbed diff & P-value \\
\hline ML displacement $(\mathrm{mm})$ & $0.22 \pm 0.76$ & $0.30 \pm 0.74$ & $0.02^{*}$ \\
\hline AP displacement $(\mathrm{mm})$ & $0.10 \pm 0.51$ & $0.55 \pm 0.54$ & $<0.001^{*}$ \\
\hline Path length $(\mathrm{m})$ & $0.35 \pm 0.21$ & $2.47 \pm 0.82$ & $0.03^{*}$ \\
\hline ML velocity $(\mathrm{mm} / \mathrm{s})$ & $0.12 \pm 1.04$ & $0.47 \pm 1.48$ & 0.11 \\
\hline AP velocity $(\mathrm{mm} / \mathrm{s})$ & $0.32 \pm 0.99$ & $1.40 \pm 0.73$ & $<0.001^{*}$
\end{tabular}

ML: medial-lateral, AP: anterior-posterior, *Significant at $\mathrm{p}<0.05$

\section{Discussion}

The obtained COP parameters were affected by localized plantar flexor muscles fatigue in the perturbed standing condition but not in the quiet standing. Our results demonstrated that in the perturbed stance condition, AP postural control was significantly impaired. This finding is consistent with the results of studies which showed that the plantar flexor muscles are more involved in sagittal plane movements than in movements in the other planes [4-7]. For example, Gimmon et al. found that during plantar flexor muscle fatigue, sway parameters were affected mainly in the AP direction and less in the ML direction [6].

Moreover, the results indicated that the plantar flexor muscles fatigue had no significant effects on the postural control parameters during bipedal static standing. The results showed that the muscle fatigue affected the postural control mainly in the more difficult standing tasks, whereas it had negligible effects during quiet standing. These findings are consistent with the results published by Adlerton et al., who reported that isometric exercise-induced fatigue of the plantar flexor muscles did not affect postural control during quiet standing [18]. Nevertheless, several studies have reported that plantar flexor muscle fatigue impaired postural stability in static conditions $[6,9,16$, 17]. Overall, most studies found impaired postural control mainly when the individuals were not able to use visual sensory inputs, somatosensory inputs from the ankle or haptic cues from the fingers in the quiet standing condition [23]. They suggested that the effect of induced fatigue in the plantar flexor muscles is greater under more challenging conditions of postural control, which is consistent with our findings [23].

Multiple sensory systems and motor synergies of the nervous system are integrated in postural control. In addition, the central nervous system (CNS) concurrently adjusts the relative contributions of sensory inputs to maintain a stable stance [24]. When fatigue is induced to the plantar flexor muscles, the CNS may be able to more readily compensate for the impaired ankle proprioception by relying on other sensory inputs, and consequently providing more reliable information to regulate body sway, as suggested by the reweighting theory [25]. Previous studies have suggested that when the muscles are fatigued, the decreased ankle joint control can be compensated by increasing the reliance on other sensory inputs (visual, plantar sole sensation and vestibular) or other motor outputs $[5,16$, 26]. Paillard recently suggested that proprioceptive information from peripheral structures in the ankle joints was discarded by the postural regulatory system at a central level, and that this strategy facilitates the use of the sensory information from the peripheral structures of other joints [26]. This was further supported by Pinsault and Vuillerme, who found that during quiet standing after plantar flexor muscle fatigue, young male college students relied more on the vestibular information and somatosensory inputs from the neck for postural control [23].

When the plantar flexor muscles are fatigued, the common drive of motor units of previously inactive muscles around the ankle joint increases and results in more joint stiff- 
ness through the co-activation of antagonist muscles or the activation of medial-lateral stabilizers in the ankle (ankle invertors and evertors) to reduce postural sway $[18,26]$. The participants in our study were young and healthy, therefore a more rigid strategy may have been used to maintain their stability. The use of a simple coordination pattern in younger adults during quiet standing may be associated with sufficient torque production by the ankle muscles. However, this hypothesis is not always supported by the results obtained under perturbation conditions. During double-limb stance, information from plantar flexor muscles may not be essential to determine COP position, and the effect of other muscles or joints may be more important.

Another aim of the present study was to investigate the effects of muscle fatigue on postural control after perturbations. According to our findings, sway was significantly greater in the AP direction after muscle fatigue compared to the non-fatigued condition. These results are in agreement with the findings of Boyas et al., who reported that differences in postural control were seen only in a more complex task [7]. In the other words, we hypothesized that under more difficult and challenging conditions in the postural task, the effect of fatigue on the ankle joint would be greater. The increased COP displacement following muscle fatigue may be the result of changes in the proprioceptive postural control strategy, which in turn may reflect corrective reactions in other muscles or joints. Adlerton et al. reported that after plantar flexor muscle fatigue, the postural control strategy changed from ankle to hip [27]. This was also supported by the observations of Salavati et al., who found that during dynamic balance tests, their participants (healthy men) used a hip strategy to maintain stability, especially after the fatigue protocol [17]. Of course, further kinematic analyses are needed in order to determine the effects of localized muscle fatigue on the segmental strategies used to maintain postural control.
Our comparison of the effects of plantar flexor muscle fatigue in quiet and perturbed stance conditions showed that reliance on proprioceptive signals from the fatigued joint decreased when quiet standing was perturbed. In the quiet standing condition, the CNS can effectively control posture even if the calf muscles are fatigued [18]. Small oscillations in quiet standing probably stimulate stretch reflex responses in the ankle joints, which are not controlled by high-level centers. In fact, in the quiet standing condition, anti-gravity muscles in the lower limbs reportedly require little activity [28]. Moreover, according to the re-weighing theory, the postural control system can compensate for the effects of fatigue. Nevertheless, it appears that under more complex conditions, the muscular response to perturbation is beyond the stretch reflex, and as a result the high-level centers are engaged [29]. Furthermore, intense fatigue is believed to affect the central motor drive as well as the motor output [30]. In the present study, the muscles were fatigued by high-intensity isometric contractions, and consequently the effects of fatigue appear to be related mostly to central mechanisms of fatigue. Therefore, the fatigue protocol apparently changed the central processing of the orders related to muscle responses, and consequently changed the motor strategies. Under difficult conditions such as dynamic postural activities, the attentional demands on the postural control system for regulating and correcting postural responses are probably greater [31]. On the other hand, in the static condition the decrease in ankle joint proprioception may not be sufficient to affect postural control when other sensory information is available. However, the presence of this information is not sufficient to compensate for the effects of fatigue induced in and around the ankles on the control of perturbed stance.

By assessing the effects of muscle fatigue on postural control regulation in two different conditions, additional information has been obtained on how sensorimotor integra- 
tion optimizes postural control in humans. We propose three hypotheses to explain our findings: (1) the CNS uses different combinations of sensory and motor strategies to maintain postural control depending on the difficulty of the postural task, (2) external perturbations increase the attentional demands for regulating body sway, and (3) ankle proprioception plays a smaller role in movement strategies after plantar flexor muscle fatigue, especially in postural tasks carried out under perturbed conditions.

There were several limitations in the current study. The participants were all young women who were not good and complete indicators for the community subjects. Moreover, the fatigue was not measured with an accurate method such as electromyography. In addition, joint kinematics and ankle proprioception have not been evaluated in this investigation. However, this study evaluated the effects of plantar flexor muscles fatigue on the postural control during a challenging condition (unexpected perturbation), i.e. in an experimental situation that has not been used in many previous studies.

\section{Conclusion}

The present experiments showed that perturbation can increase postural sway when the plantar flexor muscles are fatigued. In the fatigue condition, the CNS may decrease its reliance on proprioceptive information from the ankles in more difficult postural tasks, and the use of other sensory inputs as well as motor outputs to provide a more reliable strategy to regulate postural sway.

\section{Acknowledgment}

The present article is based on the thesis prepared by Zahra Amiri with the financial support from the Vice-Chancellor for Research at Shiraz University of Medical Sciences (Grant no. 5682). We thank the Research Improvement Center of Shiraz University of Medical Sciences for their support, and K. Shashok
(AuthorAID in the Eastern Mediterranean) and Ms. A. Keivanshekouh for improving the use of English in the manuscript.

\section{Conflict of Interest}

None

\section{References}

1. Jacobs JV, Horak FB. Cortical control of postural responses. J Neural Transm (Vienna). 2007;114:1339-48. doi: 10.1007/s00702-0070657-0. PubMed PMID: 17393068; PubMed Central PMCID: PMC4382099.

2. Horak FB, Henry SM, Shumway-Cook A. Postural perturbations: new insights for treatment of balance disorders. Phys Ther. 1997;77:517-33. PubMed PMID: 9149762.

3. Vuillerme N, Danion F, Forestier N, Nougier V. Postural sway under muscle vibration and muscle fatigue in humans. Neurosci Lett. 2002;333:131-5. PubMed PMID: 12419498.

4. Ochsendorf DT, Mattacola CG, Arnold BL. Effect of orthotics on postural sway after fatigue of the plantar flexors and dorsiflexors. J Athl Train. 2000;35:26-30. PubMed PMID: 16558604; PubMed Central PMCID: PMC1323434.

5. Gribble PA, Hertel J. Effect of lower-extremity muscle fatigue on postural control. Arch Phys Med Rehabil. 2004;85:589-92. PubMed PMID: 15083434.

6. Gimmon Y, Riemer R, Oddsson L, Melzer I. The effect of plantar flexor muscle fatigue on postural control. J Electromyogr Kinesiol. 2011;21:9228. doi: 10.1016/j.jelekin.2011.08.005. PubMed PMID: 21917475.

7. Boyas S, Remaud A, Bisson EJ, Cadieux S, Morel B, Bilodeau M. Impairment in postural control is greater when ankle plantarflexors and dorsiflexors are fatigued simultaneously than when fatigued separately. Gait Posture. 2011;34:2549. doi: 10.1016/j.gaitpost.2011.05.009. PubMed PMID: 21646020.

8. Ivanenko YP, Talis VL, Kazennikov OV. Support stability influences postural responses to muscle vibration in humans. Eur $\mathrm{J}$ Neurosci. 1999;11:647-54. PubMed PMID: 10051765.

9. Caron 0 . Effects of local fatigue of the lower limbs on postural control and postural stability in standing posture. Neurosci Lett. 2003;340:836. PubMed PMID: 12668242. 
10. Harkins KM, Mattacola CG, Uhl TL, Malone TR, McCrory JL. Effects of 2 ankle fatigue models on the duration of postural stability dysfunction. J Athl Train. 2005;40:191-4. PubMed PMID: 16284640; PubMed Central PMCID: PMC1250260.

11. Kent-Braun JA. Central and peripheral contributions to muscle fatigue in humans during sustained maximal effort. Eur J Appl Physiol Occup Physiol. 1999;80:57-63. doi: 10.1007/ s004210050558. PubMed PMID: 10367724.

12. Baker AJ, Kostov KG, Miller RG, Weiner MW. Slow force recovery after long-duration exercise: metabolic and activation factors in muscle fatigue. J Appl Physiol (1985). 1993;74:2294-300. doi: 10.1152/jappl.1993.74.5.2294. PubMed PMID: 8335559.

13. Wilder DG, Aleksiev AR, Magnusson ML, Pope $\mathrm{MH}$, Spratt KF, Goel VK. Muscular response to sudden load. A tool to evaluate fatigue and rehabilitation. Spine (Phila Pa 1976). 1996;21:262839. PubMed PMID: 9045348.

14. Granacher U, Gruber M, Forderer D, Strass D, Gollhofer A. Effects of ankle fatigue on functional reflex activity during gait perturbations in young and elderly men. Gait Posture. 2010;32:107-12. doi: 10.1016/j.gaitpost.2010.03.016. PubMed PMID: 20434345.

15. Enoka RM. Muscle fatigue--from motor units to clinical symptoms. J Biomech. 2012;45:427-33. doi: 10.1016/j.jbiomech.2011.11.047. PubMed PMID: 22197185.

16. Vuillerme N, Burdet C, Isableu B, Demetz S. The magnitude of the effect of calf muscles fatigue on postural control during bipedal quiet standing with vision depends on the eye-visual target distance. Gait Posture. 2006;24:169-72. doi: 10.1016/j.gaitpost.2005.07.011. PubMed PMID: 16226030.

17. Salavati M, Moghadam M, Ebrahimi I, Arab AM. Changes in postural stability with fatigue of lower extremity frontal and sagittal plane movers. Gait Posture. 2007;26:214-8. doi: 10.1016/j.gaitpost.2006.09.001. PubMed PMID: 17049237.

18. Adlerton AK, Moritz U. Does calf-muscle fatigue affect standing balance? Scand J Med Sci Sports. 1996;6:211-5. PubMed PMID: 8896093.

19. Caron 0 . Is there interaction between vision and local fatigue of the lower limbs on postural control and postural stability in human posture? Neurosci Lett. 2004;363:18-21. Doi: 10.1016/ s0304-3940(04)00338-6.

20. Gribble PA, Hertel J. Effect of hip and ankle muscle fatigue on unipedal postural control. $J$ Electromyogr Kinesiol. 2004;14:641-6. doi: 10.1016/j.jelekin.2004.05.001. PubMed PMID: 15491838.

21. Boyas S, Hajj M, Bilodeau M. Influence of ankle plantarflexor fatigue on postural sway, lower limb articular angles, and postural strategies during unipedal quiet standing. Gait Posture. 2013;37:547-51. doi: 10.1016/j.gaitpost.2012.09.014. PubMed PMID: 23102672.

22. Kennedy A, Hug F, Bilodeau M, Sveistrup $H$, Guevel A. Neuromuscular fatigue induced by alternating isometric contractions of the ankle plantar and dorsiflexors. J Electromyogr Kinesiol. 2011;21:471-7. doi: 10.1016/j.jelekin.2011.02.001. PubMed PMID: 21376628.

23. Pinsault N, Vuillerme N. Differential postural effects of plantar-flexor muscle fatigue under normal, altered and improved vestibular and neck somatosensory conditions. Exp Brain Res. 2008;191:99-107. doi: 10.1007/s00221-0081500-z. PubMed PMID: 18663436.

24. Vuillerme N, Pinsault N. Re-weighting of somatosensory inputs from the foot and the ankle for controlling posture during quiet standing following trunk extensor muscles fatigue. Exp Brain Res. 2007;183:323-7. doi: 10.1007/ s00221-007-1047-4. PubMed PMID: 17643234.

25. Janssens L, Brumagne S, Polspoel K, Troosters T, McConnell A. The effect of inspiratory muscles fatigue on postural control in people with and without recurrent low back pain. Spine (Phila Pa 1976). 2010;35:1088-94. doi: 10.1097/ BRS.0b013e3181bee5c3. PubMed PMID: 20393397.

26. Paillard T. Effects of general and local fatigue on postural control: a review. Neurosci Biobehav Rev. 2012;36:162-76. doi: 10.1016/j.neubiorev.2011.05.009. PubMed PMID: 21645543.

27. Adlerton AK, Moritz U, Moe-Nilssen R. Forceplate and accelerometer measures for evaluating the effect of muscle fatigue on postural control during one-legged stance. Physiother Res Int. 2003;8:187-99. Doi: 10.1002/pri.289.

28. Panzer VP, Bandinelli S, Hallett M. Biomechanical assessment of quiet standing and changes associated with aging. Arch Phys Med Rehabil. 1995;76:151-7. PubMed PMID: 7848073.

29. Winter DA. Human balance and posture con- 
trol during standing and walking. Gait Posture. 1995;3:193-214. Doi: 10.1016/09666362(96)82849-9.

30. Amann M, Dempsey JA. Locomotor muscle fatigue modifies central motor drive in healthy humans and imposes a limitation to exercise performance. J Physiol. 2008;586:161-73. doi: 10.1113/jphysiol.2007.141838. PubMed
PMID: 17962334; PubMed Central PMCID: PMC2375542.

31. Bisson EJ, McEwen D, Lajoie Y, Bilodeau M. Effects of ankle and hip muscle fatigue on postural sway and attentional demands during unipedal stance. Gait Posture. 2011;33:83-7. doi: 10.1016/j.gaitpost.2010.10.001. PubMed PMID: 21050763. 